\begin{tabular}{|c|c|c|}
\hline PKS & Revista de GEOGRAFIA & OJS \\
\hline $\begin{array}{l}\text { PUBLIC } \\
\text { KNOWLEDGE } \\
\text { PROJECT }\end{array}$ & $\begin{array}{c}\text { (RECIFE) } \\
\text { http://www.revista.ufpe.br/revistageografia }\end{array}$ & $\begin{array}{l}\text { OPEN } \\
\text { JOURNAL } \\
\text { SYSTEMS }\end{array}$ \\
\hline
\end{tabular}

\title{
INICIATIVA DE MAPEO LIBRE DE EXPERIENCIAS DE AGRICULTURA URBANA EN BOGOTÁ Y SUS ALREDEDORES, “AGROECOBOGOTA” (2014-2016).
}

\author{
Kharen Viviana Pinilla Guerrero ${ }^{1}$ \\ ${ }^{1}$ Profesora de Geografía de la Universidad de Cundinamarca - Colómbia. Email: .kvpinillag@unal.edu.co
}

Artigo recebido em 16/03/2017 e aceito em 21/06/2017

\begin{abstract}
RESUMEN
Este artículo muestra el origen, antecedentes, bases teóricas, herramientas de funcionamiento y posibilidades de proyección que tiene la plataforma de mapeo en tiempo real agroecobogotá.crowdmap.com. La iniciativa fue creada gracias a la sombrilla tecnológica de la corporación Keniana Ushahidi e impulsada hace aproximadamente tres años por estudiantes de posgrado y pregrado de la Universidad Nacional de Colombia, la Universidad Pedagógica Nacional y de las Universidades alemanas de Gotinga y Hamburgo ${ }^{1}$. Se ha mantenido vigente gracias a la participación de las ciudadanías interesadas en el tema agroecológico de las diferentes zonas urbanas de Bogotá y sus alrededores. La plataforma hasta el momento no ha contado con ningún respaldo institucional directo. El texto está dividido en cinco partes. En la primera recogeremos un poco sobre los lineamientos generales que delimitaron el nacimiento de Ushahidi. En la segunda relacionaremos antecedentes importantes vinculados con el mapeo participativo en línea con la tecnología crowdmap a escala mundial. En la tercera hablaremos sobre el avance teórico de la geografía dentro de la ciencias humanas y la cartografía social en línea como bases teóricas potenciadoras del empoderamiento ciudadano. En la cuarta describiremos las potencialidades funcionales que tiene la plataforma. En la quinta se darán algunas conclusiones y recomendaciones relacionadas con los puntos mapeados hasta el momento en la plataforma.
\end{abstract}

Palabras clave: Geografía, Cartografía Social en Línea, Participación Ciudadana.

\section{INICIATIVA DE MAPEAMENTO LIVRE DE EXPERIENCIAS DE AGRICULTURA URBANA EM BOGOTÁ E SEUS ARREDORES, "AGROECOBOGOTA" (2014-2016).}

\section{RESUMO}

Este artigo mostra a origem, história, fundamentos teóricos, ferramentas de trabalho e possibilidades de projeção que tem em tempo real a plataforma de mapeamento agroecobogotá.crowdmap.com. A iniciativa foi criada graças ao projeto tecnológico da corporação queniana Ushahidi e levado a cabo cerca de três anos atrás por estudantes de graduação da Universidade Nacional da Colômbia, Universidade Pedagógica Nacional e da Universidade alemã de Goettingen e Hamburgo. Ele foi mantido vivo graças à participação de interessados na

\footnotetext{
${ }^{1}$ Las personas que pertenecemos a esta iniciativa somos: Kharen Pinilla Magíster de la Universidad de Cundinamarca, Julián Ramírez, Magíster de la Universidad Nacional de Colombia. Jennifer Rodríguez Franco Magíster de la Universidad de Gottinga, Alemania. Birgit Hoinle, Estudiante de Doctorado de la Universidad de Hamburgo, Alemania. Fabián Montejo, estudiante Geografía de la Universidad Nacional de Colombia. Sarah Pinilla estudiante de licenciatura en Psicología y Pedagogía de la Universidad Pedagógica Nacional.
} 
questão das cidadanias agro-ecológicas de diferentes áreas urbanas de Bogotá e seus arredores. A plataforma até agora não teve qualquer apoio institucional direta. Neste sentido este artigo está dividido em cinco partes. No primeiro nós trataremos um pouco sobre as orientações gerais delineadas e o nascimento de Ushahidi. A segunda vai relacionar importante fundo vinculado com o mapeamento participativo em linha com a tecnologia Crowdmap em escala mundial. O terceiro irá discutir o avanço teórico da geografia no âmbito das ciências humanas e mapeamento on-line social como bases teóricas potenciadoras de empoderamento dos cidadãos. No quarto descrevemos as potencialidades funcionais da plataforma. No quinto discorremos sobre algumas conclusões e recomendações relativos aos pontos mapeados até agora da plataforma.

Palavras-chave: Geografia, Cartografia Social, Participação cidadã.

\section{¡LOS SENSORES HUMANOS EN INTERACCIÓN!}

La iniciativa de mapeo en tiempo real de 1) Alimentos y productos naturales, 2) Semillas e insumosorgánicos y, 3) Educación agroecológica ypermacultural: Mapeo Agroecobogota nació por la sombrilla tecnológica que ofrece la corporación tecnológica Keniana Ushahidi, que significa testimonio en swahili lengua oficial en tres países africanos: Kenia, Uganda y Tanzania.

Ushahidi se dio al mundo en medio de la fragilidad de un Estado en caos por la violencia postelectoral desatada en el 2007 y 2008 en Kenia, África. Se calcularon un total de 1.300 víctimas entre heridos, muertos y comerciantes quebrados por los saqueos multitudinariosen medio dela efervescencia que producía una toma de poder controversial (Ushahidi, 2012). La dificultad para obtener información verídica de las múltiples violaciones a los derechos civiles que estaban ocurriendo simultáneamente en varias ciudades del país africano, hizo que el aporte de las mismas ciudadanías fuera la única manera en la que los organismos de socorro lograran dimensionar la magnitud de la tragedia. El mapeo que generaba Ushahidi por medio de su plataforma web generaba un rastreo en tiempo real del lugar exacto en donde estaba ocurriendo el evento de violencia, junto con el contacto directopara facilitar la comunicación y el punto de referencia para el desplazamientodel cuerpo médico que atendía las víctimas. La participación ciudadana fue el elemento más valioso de denuncia y control social; retirando la necesidad de trámites y el peligro inminente de las denuncias directas.

Las ciudadanías mediante sus dispositivos móviles pueden denunciar mediante texto, imagen y vídeo las diferentes formas de violencia ocurrida, la cantidad de muertes, saqueos, maltratos y demás violaciones a los derechos humanos en tiempo real, haciendo las veces de sensores humanos del territorio. Al respecto se afirma: 
El Presidente de Kenia MwaiKibaki fue anunciado el 30 de diciembre; sin embargo, el candidato de la oposición RailaOdinga afirmó que las papeletas fueron manipuladas. Miembros de Luo de OdingaTribe se enfurecieron, provocando tensiones de décadas con la tribu Kikuyu de Kibaki. El caos provocó un período turbulento en la historia de Kenia, lo que llevó a activistas kenianos a enviar una súplica en su blog KenyanPundit (...). Esta simple consulta del 3 de enero de 2008 inspiró a un grupo de bloggers a colaborar durante el fin de semana para convertir su sueño en una realidad. El 9 de enero, Ushahidi, que significa "testigo o testimonio" en swahili, nació, lo que permitió a los kenianos y personas de todo el mundo reportar y Violencia vía SMS o la web.

(Ushahidi, 2012)

La información canalizada mostró la crudeza de la muerte, los saqueos y ataques violentossufridos en varias ciudades Kenianas. La creación del mapa vivo fue clave parala elaboración de los informes de denuncia frente a la evidente guerra civil que se desataba en Kenia. (Herreño, 2012)

Ushahidi se convirtió en un éxito rotundo y, a partir de allí, se han creado varias plataformas interactivas que aprovechan las potencialidades para mapear en tiempo real diferentes fenómenos espaciales. Resaltamos que para la creación de estas plataformas existen dos métodos diferentes, que van de acuerdo al público que las demanda. Para los desarrolladores avanzados se puso a disposición la base de datos libre con código abierto (ODbL) en donde se usa un lenguaje de programación (sea para Windows o Linux) con posibilidad de mejora colectiva continua, conservando los derechos de autor de los generadores de la información, es decir facilitando la creación de conocimiento colectivo en web y el licenciamiento libre ${ }^{2}$. Para las personas que no dominamos lenguajes de programación, la extensión liberó la base de código general que permite la creación automática de plataformas estándar, acompañadas con un nivel de amplio de personalización web.

\section{ANTECEDENTES DE MAPEOS COLABORATIVOS CON LA TECNOLOGÍA CROWDMAP}

Al realizar el rastreo en la web sobre mapeos colaborativos que han empleado la tecnología Ushahidi-crowdmap, nos hemos dado cuenta que existen seis temas centrales de seguimiento en tiempo real: 1) Mapeos de denuncia por manifestaciones de violencia; 2)

\footnotetext{
${ }^{2}$ En cuanto al licenciamiento libre, aclaramos que la plataforma por defecto dispone de cuatro grupos de bases cartográficas a escala mundial en las que se incluye a Google Earth, Open Street Maps, Bing y Esri.
} 
Mapeos de control ciudadano frente a la dinámica migratoria; 3) Mapeos de control ciudadano frente a la dinámica urbana; 4) Mapeos relacionados con manifestaciones de coyunturas políticas; 5) Mapeos relacionados con experiencias en educación y 6) Mapeos relacionados con dinámicas medioambientales (Tanto de cuidado del entorno como de seguimiento a eventos naturales adversos). Organizamos la información de acuerdo a la ubicación geográfica, objetivo general del mapeo, las categorías presentes y la dirección web en donde se puede visualizar la herramienta en línea.

La escala de mapeo de cada plataforma es mundial, pero casualmente cada una tiene una referencia centralizada a un solo país o ciudad, solo unas pocas han tenido trascendencia de escala geográfica. Esto probablemente se debe a que es una iniciativa incipiente.

En cuanto a los mapeos de denuncia por manifestaciones de violencia encontramos relacionados los siguientes países: Territorio ocupado Palestino, Israel. Syria. México. España, Brasil y Sudáfrica. El objetivo de los sensores humanos ha sido la visibilización de las víctimas del conflicto. En el territorio ocupado Palestino e Israel es escalofriante ver los reportes de menores de edad detenidos, lesionados y desaparecidos, se narran los sucesos ocurridos y se clasifica la información de acuerdo con el responsable del hecho, sea un grupo armado palestino, autoridad palestina, militar Israelí o colono Israelí. No hay archivos de vídeo en la plataforma, solo muestra un archivo de imagen de SameerAwad de 16 años, quien murió a causa de munición real por parte de un militar Israelí.

En el caso de Syria la situación es aún más impactante, puesto que es el mapeo de violencia sexual en medio de la guerra. Los reportes se han mantenido desde el 2011 hasta el cuatro de febrero de 2016. Las categorías más mapeadas han sido 1) Forzado vaginal, anal u otra entrada a manos de las fuerzas gubernamentales. -con ciento noventa y ocho reportes de doscientos cuarenta y seis reportes en total- y; 2) Consecuencias de la violencia sexual. -con ciento cincuenta de doscientos cuarenta y seis reportes-; lo que más se nota es la ansiedad o depresión como consecuencia traumática de la violencia sexual, seguida por muerte con signos de violencia sexual. 
Cuadro 1. Mapeos de denuncia por manifestaciones de violencia, 2016.

\begin{tabular}{|c|c|c|c|}
\hline UBICACIÓN GEOGRÁFICA & \begin{tabular}{|l|} 
OBJETIVO DEL MAPEO \\
\end{tabular} & CATEGORÍAS & DIRECCIÓN WEB \\
\hline $\begin{array}{c}\text { Territorio ocupado Pales tino - } \\
\text { Israel }\end{array}$ & $\begin{array}{l}\text { Mapeo acciones violentas dirigidas } \\
\text { hacia los menores de edad en el } \\
\text { Territorio ocupado Palestino e Israel. }\end{array}$ & $\begin{array}{l}\text { 1) Delicado } \quad \text { - 2) } \\
\text { Lesionado } \quad \text { - 3) Detenido. }\end{array}$ & http://crowdmap.dci-palestine.org/ \\
\hline México & $\begin{array}{c}\text { Mapeo de los lugares de desaparición } \\
\text { y muerte de mujeres y niñas en } \\
\text { México. }\end{array}$ & $\begin{array}{l}\text { 1) Feminicidios; 2) } \\
\text { Desapariciones. }\end{array}$ & $\begin{array}{l}\text { https://www.google.com/maps/d/u/0/viewe } \\
\text { r?mid=174IjBzP- } \\
\text { fl_6wpRHg5pkGS2egE\&ll=19.37539730 } \\
6733415.99 .12165745000004 \& z=11\end{array}$ \\
\hline México y Brasil & $\begin{array}{c}\text { Mapeo de agresiones a periodístas en } \\
\text { América Latina. }\end{array}$ & $\begin{array}{l}\text { 1) Agresión física; 2) Agresión } \\
\text { jurídica; 3) Agresión } \\
\text { psicológica; 4) Agresión digital. }\end{array}$ & $\begin{array}{l}\text { https://periodistasenriesgo.crowdmap.co } \\
\underline{\mathrm{m} /}\end{array}$ \\
\hline México & $\begin{array}{l}\text { Mapeo de víctimas del conflicto en } \\
\text { México. }\end{array}$ & $\begin{array}{l}\text { 1) Víctimas No son cifras; 2) } \\
\text { Propuestas de cambio; } 3 \text { ) } \\
\text { Acciones cívicas. }\end{array}$ & https://pazyjusticia.crowdmap.com \\
\hline
\end{tabular}

Fuente. Elaboración propia.

En México hay dos mapeos relacionados con denuncias por violencia. El primero vinculado con la muerte y desaparición de mujeres y niñas en el Estado de México ${ }^{3}$. Y en el segundo plantea el mapeo de agresiones hechas a periodistas en América Latina las cuales se han concentrado en México y un poco en Brasil. En España por su parte ha habido un fenómeno interesante de seguimiento en tiempo real de las incidencias territoriales de la huelga general realizada en el país el pasado 29 de septiembre de 2010. Mientras que en

${ }^{3}$ Frente a los feminicidios resaltamos que "La mayoría de los feminicidios y sus responsables existen en un entorno marcado por distintos tipos de marginación, lo cual quizá sugiera una "protesta sub-política y personal de carácter conservador" (Leyton, 2005:43) que trata de mostrar el desacuerdo frente a la construcción de las mujeres como sujetos. Una especie de rebelión contra un orden social que los ha despojado no sólo de la capacidad de ser proveedores, autoridad, cabeza de familia, sino de usufructuar los beneficios de las formas tradicionales de dominio frente a las mujeres". (Arteaga y Valdez, 2009) 
Sudáfrica se montó la plataforma se seguimiento a la delincuencia en la zona Jeffrey'sBays en donde se mapean las zonas donde se ha visto comportamiento sospechoso, los daños a personas, a propiedades y las víctimas relacionadas con este tipo de violencia.

Cuadro 2. Mapeos de control ciudadano frente a la dinámica migratoria.

\begin{tabular}{|c|c|c|c|}
\hline Tunez e Italia. & $\begin{array}{c}\text { Mapeo de las muertes de los migrantes y } \\
\text { las violaciónes de sus derechos en el las } \\
\text { fronteras marítimas de la Unión Europea. }\end{array}$ & $\begin{array}{c}\text { 1) Patralla marítima; 2) } \\
\text { Muerto; 5) Angustiado; 6) } \\
\text { Testimonio; 7) Desaparecido; 8) } \\
\text { Operación de rescate. }\end{array}$ & https://watchthemed.crowdmap.com/ \\
\hline España & $\begin{array}{l}\text { Mapeo de la concentración para lograr el } \\
\text { cierre de los Centros de Internamiento de } \\
\text { Extranjeros }\end{array}$ & $\begin{array}{c}\text { 1) Encuentros y jornadas; 2) } \\
\text { Concentraciones; 3) Informes; 4) } \\
\text { Deportaciones suspendidas. }\end{array}$ & https://stopcies.crowdmap.com \\
\hline
\end{tabular}

Fuente. Elaboración propia.

Cuadro 3. Mapeos de control ciudadano frente a la dinámica urbana.

\begin{tabular}{|c|c|c|l|}
\hline Ecuador & $\begin{array}{c}\text { Mapeo de control ciudadano frente a los } \\
\text { problemas urbanos en la ciudad de } \\
\text { Cuenca. }\end{array}$ & $\begin{array}{c}\text { 1) Movilidad, 2) Medio ambiente; } \\
\text { 3) Equipamiento urbano; 4) } \\
\text { Seguridad; 5) Salud; 6) } \\
\text { Participación Ciudadana. }\end{array}$ & https://cuencavisible.crowdmap.com/ \\
\hline Argentina & $\begin{array}{c}\text { Mapeo de los problemas ambientales } \\
\text { ocurridos en el cordón industrial del Gran } \\
\text { Rosario. }\end{array}$ & $\begin{array}{c}\text { 1) Denuncias; 2) Actividades; 3) } \\
\text { Propuestas. }\end{array}$ & $\begin{array}{c}\text { https://cordonindustrialrosario.crowdmap } \\
\text {.com/ }\end{array}$ \\
\hline Nigeria & $\begin{array}{c}\text { Mapeo para documentar la resistencia } \\
\text { ciudadana ante la situación del tráfico de } \\
\text { transporte en el Estado de Lagos. }\end{array}$ & $\begin{array}{c}\text { 1) Accidente automovilístico; 2) } \\
\text { Lesión; 3) Muerte; 4) Advertencia } \\
\text { temprana. }\end{array}$ & https://lagostraffic.crowdmap.com/ \\
\hline Colombia & $\begin{array}{c}\text { Mapeo de problemas relacionados con el } \\
\text { acueducto y alcantarillado del altiplano } \\
\text { cundiboyacesce. }\end{array}$ & $\begin{array}{c}\text { 1) Acueducto; 2) Alcantarillado; 3) } \\
\text { Manejo de basuras. }\end{array}$ & https://inalambria.crowdmap.com/ \\
\hline España & $\begin{array}{c}\text { Mapeo de control ciudadano frente a los } \\
\text { problemas urbanos en la ciudad de Alcalá } \\
\text { de Henares. }\end{array}$ & $\begin{array}{c}\text { 1) Vandalismo; 2) Suciedad; 3) } \\
\text { Transporte público; 4) Vehículos } \\
\text { abandonados; 5) Ruido; 6) Jardín } \\
\text { abandonado; 7) Contenedor roto; } \\
\text { 8) Desperfectos. }\end{array}$ & https://alcaladehenares.crowdmap.com/ \\
\hline
\end{tabular}

Fuente. Elaboración propia.

En cuanto a los mapeos de control ciudadano destacamos dos categorías generales: la primera relacionada con la dinámica migratoria y la segunda con la dinámica urbana.

Los mapeos relacionados con la dinámica migratoria se concentran en la geografía del Mar Mediterráneo, especialmente en las fronteras entre los países Europeos y los Africanos en donde las garantías legales se hacen tan difusas.

Entre Túnez e Italia los reportes obedecen a la visibilización de los testimonios de las víctimas de maltrato, desaparición, muerte, presencia de patrullas marítimas, entre otros. Entre España y la Costa Norte Africana, los reportes se relacionan con una fuerte campaña política que busca el cierre de los centros de internamiento de extranjeros -establecimientos penitenciarios- y suspender las deportaciones.

En cuanto a los mapeos de control ciudadano frente a la dinámica urbana, encontramos cinco países relacionados: Ecuador, Argentina, Nigeria, Colombia y España. En todos se han querido evidenciar los denominados "problemas urbanos" relacionados con el 
tráfico de transporte terrestre, medio ambiente, seguridad, condiciones del saneamiento básico -acueducto y alcantarillado-. En todos se ha visto el deseo de incentivar la participación ciudadana para hacer propuestas de cambio frente a los problemas ocurridos.

Cuadro 4. Mapeos relacionados con manifestaciones de coyunturas políticas

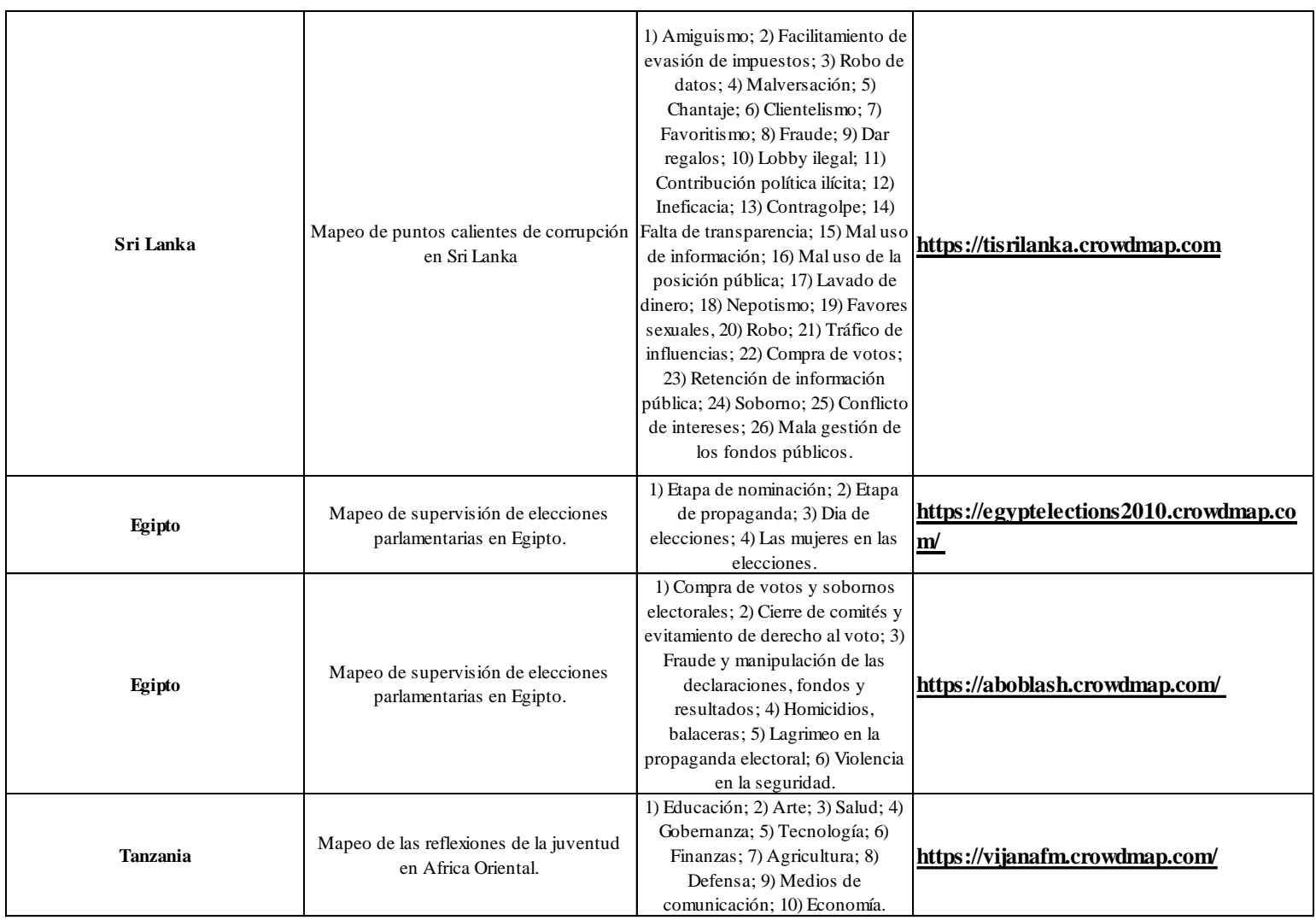

Fuente. Elaboración propia.

Las incidencias territoriales de los mapeos vinculados con coyunturas políticas tienen la facilidad de mostrar de manera muy simple y contundente las diferentes caras de las soluciones democráticas relacionadas con el ejercicio del poder. Una de las plataformas más interesantes que analizamos fue el mapeo de puntos calientes de corrupción en Sri Lanka, con un total de veintiséis categorías, muy reveladoras cada una de ellas (cuadro 4). Los reportes más concentrados han sido los relacionados con: falta de transparencia, mal uso de la posición pública, el favoritismo y la ineficiencia de los gobernantes del país asiático.

En Egipto hay dos mapeos relacionados con la erupción de las potencialidades de las redes sociales en la organización política del país. En ambas plataformas el fin era la supervisión de las ciudadanías frente a las elecciones parlamentarias hechas en 2010. La primera muestra simplemente los hechos ocurridos en las nominaciones, procesos de campaña, el día de las elecciones y la posición de la mujer. En la segunda se nota un cambio 
impresionante porque se establecen seis categorías que muestran la crudeza de la violencia ejecutada contra la población en este ejercicio democrático. Las categorías más mapeadas son: fraude y manipulación de las declaraciones, fondos y resultados y, lagrimeo en la campaña electoral.

En Tanzania, por su parte, se hizo un ejercicio interesante frente a la posición política de las juventudes del país. Se mapearon las reflexiones que tenían sobre la educación, la salud, la economía, los medios de comunicación, entre otros asuntos relevantes del país.

La educación como ejercicio de memoria colectiva también ha sido la causa de mapeos colectivos. En estos mapeos lo interesante es que han transcendido fronteras internacionales en su mayoría y tratan temas profundamente esperanzadores.

En Italia surgieron dos mapeos, el primero relacionado con los sitios web escolares discriminadas por el tipo de software libre empleado, el segundo tiene que ver con la expedición del buque oceanográfico "Urania" en los alrededores del Mar Mediterráneo, en éste se reportó información relacionada con mediciones ecológicas, mediciones espectro radiométricas, diario de viaje y archivos multimedia.

En el continente Asiático la comunidad LGTBI montó su plataforma de mapeo para afianzar sus condiciones de participación y entorno legal en el mundo, cuenta con categorías relacionadas con discriminación, violencia, acoso verbal, empleo, salud, orgullo y concentraciones masivas, entre otras.

En Colombia hay una plataforma relacionada con la construcción de memoria colectiva. El mapeo fue dirigido por el Movimiento de Víctimas de Crímenes del Estado (MOVICE) compila las dinámicas geo-históricas del país desde la década de los $20^{\circ}$ hasta el 2014. Tiene categorías relacionadas con temas que han hecho parte de la historia del conflicto armado determinado por los impulsos y las restricciones en el Territorio. Las más mapeadas han sido la violación a los Derechos Humanos, los modelos económicos impuestos, las consecuencias colectivas del conflicto armado y las acciones reivindicativas que incluye el seguimiento histórico de las manifestaciones, huelgas, marchas y paros durante gran parte del siglo XX hasta el 2014. Es una herramienta muy interesante porque liga la historia y la geografía de Colombia.

Las cuatro plataformas restantes tienen escala global y relacionan pequeñas revoluciones hechas en el mundo en el ámbito educativo, la primera se refiere a las acciones heróicas de los jóvenes en el mundo, la cual hace parte de una gran campaña de visibilización de las pequeñas acciones de solidaridad del cotidiano vivir. La segunda es el mapeo de las 
experiencias de educación alternativa en el mundo. La tercera tiene que ver con las iniciativas de celebración de comunidades juveniles radiales en el mundo, a propósito del día mundial de la Radio, celebrado en el 2015. La cuarta tiene que ver con una iniciativa de registro documental del patrimonio audiovisual en el mundo, ha sido alimentada por diversos colectivos Latinoamericanos.

En cuanto a los mapeos relacionados con el medio ambiente, los podríamos dividir en dos grandes categorías: 1) cuidado del medio ambiente y; 2) seguimiento a eventos naturales adversos. Los países involucrados son Colombia. Argentina. Bolivia. Ecuador. México. España. Países Bajos. Estados Unidos. Inglaterra. Filipinas. Alemania. Canarias. India. Paquistán y Canadá. 
Revista de Geografia (Recife) V. 34, No. 2, 2017

Cuadro 5. Mapeos relacionados con educación

\begin{tabular}{|c|c|c|c|}
\hline Italia & Mapeo de sitios web escolares & $\begin{array}{l}\text { 1) Drupal; 2) Joomla; 3) Plone; 4) } \\
\text { Wordpress; 5) Harmonia; 6) Sitio } \\
\text { escolástico. } \\
\end{array}$ & https://porteapertesulweb.crowdmap.com/ \\
\hline Italia & $\begin{array}{l}\text { Mapeo de la información recogida en la } \\
\text { expedición cientítfica del buque } \\
\text { oceanográfico Urania en el Mar Adriático. }\end{array}$ & $\begin{array}{l}\text { 1) Diario de viaje; 2) Medicions } \\
\text { espectroradiométricas, 3) } \\
\text { Mediciones ecológicas; 4) } \\
\text { Mediciones de olas; 5) Multimedia. }\end{array}$ & https://uraniaexpedition.crowdmap.com/ \\
\hline Mundo & $\begin{array}{l}\text { Mapeo de las acciones heróicas de los } \\
\text { jóvenes en el mundo. }\end{array}$ & $\begin{array}{c}\text { 1) Centros de colaboración; 2) } \\
\text { Medios y periodistas; 3) Millenials } \\
\text { e innovaciones; 4) Juveniles; 5) } \\
\text { Millenials y salud; 6) Millenials y } \\
\text { aprendizaje; 7) Empoderamiento de } \\
\text { la mujer del milenio. }\end{array}$ & https://socialbusiness.crowdmap.com/ \\
\hline Países del contienente Asiático & $\begin{array}{l}\text { Mapeo de las condiones de participación } \\
\text { y el entorno legal y social de las } \\
\text { Lesbianas Gays, Bisexuales y } \\
\text { Transexuales de Asia. }\end{array}$ & $\begin{array}{l}\text { 1) Discriminación; 2) Violencia; 3) } \\
\text { Acoso verbal; 4) Legislación; 5) } \\
\text { Tendencias sociales; 6) } \\
\text { Movimientos positivos; 7) Orgullo } \\
\text { y concentraciones; 8) Historias } \\
\text { personales; 9) Empleo; 10) Salud; } \\
\text { 11) Religión; 12) Asuntos } \\
\text { familiares y 13) Recursos. }\end{array}$ & https://beinglgbtinasia.crowdmap.com/ \\
\hline Mundo & $\begin{array}{l}\text { Mapeo de iniciativas radiales en el día } \\
\text { mundial de la Radio, } 2015\end{array}$ & $\begin{array}{l}\text { 1) Evento oficial; 2) Evento } \\
\text { público; 3) Educación; 4) Foro de } \\
\text { discusión; 5) Producción de radio } \\
\text { juvenil. } \\
\end{array}$ & https://worldradioday2015.crowdmap.com/ \\
\hline Mundo & $\begin{array}{l}\text { Mapeo de experiencias de educación } \\
\text { alternativa en el mundo }\end{array}$ & $\begin{array}{l}\text { 1) Tipo de experiencia; 2) Tipo de } \\
\text { enfoque alternativo; 3) Tipo de } \\
\text { método pedagógico; 4) Por } \\
\text { gestión. }\end{array}$ & http://mapa.reevo.org/ \\
\hline Mundo & $\begin{array}{l}\text { Mapeo del patrimonio audiovisual para la } \\
\text { reivindicación de la momeria colectiva de } \\
\text { América Latina y el Caribe. }\end{array}$ & \begin{tabular}{|c|} 
1) Conflictos socio-ambientales y \\
territorio; 2) Medios de \\
comunicación e industria cultural; \\
3) Software libre; 4) Capitalismo y \\
procesos productivos; 5) Género y \\
antipatriarcado; 6) Diversas \\
sexualidades; 7) Derechos sexuales \\
y reproductivos; 8) \\
Criminalización, represión e \\
impunidad; 9) Movilización y \\
protesta; 8) Organización y \\
autogobierno; 9) Derechos \\
humanos y comunales; 10) \\
Migración y desplazamiento; 11) \\
Derecho a la comunicación; 12) \\
Culturas, identidades y memoria; \\
13) Infancia, juventud y adultos \\
mayores; 14) Otros mundos \\
posibles. \\
\end{tabular} & https://territorioaudiovisual.crowdmap.com/ \\
\hline Colombia & $\begin{array}{l}\text { Mapeo histórico de impulsos y } \\
\text { restricciones en el Territorio. }\end{array}$ & \begin{tabular}{|l} 
1) Acciones reivindicativas; 2) \\
Efectos colectivos del crimen; 3) \\
Expectativas y propuestas de \\
reparación; 4) Líderes de la \\
restitución de tierras asesinados \\
por el gobierno de Santos; 5) \\
Logros de verdad y justicia; 6) \\
Modelos económicos; 7) Políticas \\
de impunidad; 8) Proyectos de \\
vida; 9) Responsables; 10) \\
Titulaciones; 11) Violación a los \\
Derechos Humanos. \\
\end{tabular} & https://conlospiesporlatierra.crowdmap.com/ \\
\hline
\end{tabular}

Fuente. Elaboración propia.

Frente a la primera categoría encontramos la iniciativa de mapeo de experiencias de agricultura urbana en Bogotá y sus alrededores, de la cual profundizaremos más adelante. La segunda relaciona experiencias de permacultura en Argentina, en donde se relacionan temas como bio-construcción, mingas, trabajo voluntario, eco-aldeas, entre otros. En España hay una iniciativa interesante de experiencias soberanía alimentaria, en donde se circulan pequeñas economías de mercado campesino, consumo responsable, huertas urbanas y la ubicación de organizaciones que defienden la soberanía alimentaria. También resaltamos la experiencia de mapeo de la adopción del Hexayurt como opción de vivienda alternativo, al 
parecer popular en Inglaterra, Alemania, Islas Canarias e India. Por último en Estados Unidos montaron una plataforma para hacer seguimiento y recuperación de las cuencas bajas y los océanos del país.

En cuanto al seguimiento a eventos naturales adversos, encontramos siete plataformas. Tres de ellas nacidas en Estados Unidos como propuesta de seguimiento a Tornados, Huracanes e incendios forestales. En ellas se reportaron los daños, inundaciones y damnificados de los hechos ocurridos. Por su parte en Filipinas se creó la plataforma para medir los daños del TifonBopha, teniendo como categorías las principales vías de evacuación, los deslizamientos de tierra y las inundaciones.

En Cornwalles, Costa sur de Inglaterra, se mapeó en tiempo real los efectos de las inundaciones en las carreteras, interrupciones de las estaciones de tren, el estado de las escuelas y las sugerencias del gobierno. En contraste, Paquistán se hizo un ejercicio similar, pero las categorías fueron mucho más específicas. Se crearon once en total, relacionadas con salud, disponibilidad de agua potable, servicios disponibles, seguridad y magnitud de las inundaciones, emergencias, abrigo, seguridad, voluntarios, protección, comida y migración. Por último, para el departamento del Chocó se creó la herramienta de mapeo en tiempo real para monitorear de manera permanente las situaciones de emergencia y desastre en la zona. Se crearon variables relacionadas con brotes y epidemias, emergencias y desastres, reportes de deslizamientos, necesidades médicas y rutas de la red prestadora de servicios de salud.

Resaltamos en este apartado, que algunas de las plataformas relacionadas anteriormente han sido impulsadas por proyectos de las Naciones Unidas. Los ejemplos de mapeo dados anteriormente son muestra de la posibilidad de ver el territorio como un organismo vivo que experimenta impulsos constantes de energía: tanto de la tierra misma, como de sus habitantes. El objetivo del mapeo en tiempo real sea quizá hacer un registro de estas sincronías espacio-temporales.

En muchos de los reportes se registra material de imagen y vídeo mostrando una cartografía visual y sonora de los diferentes fenómenos espaciales. La posibilidad de entender las situaciones de conflicto en la lectura hecha por sus propias víctimas le imprime un sentido político de visibilización muy necesario para comprender las dinámicas socio-espaciales de ahora. Cada plataforma de mapeo es una perspectiva de mundo que se muestra y se potencia. 
Cuadro 6. Mapeos relacionados con dinámicas medioambientales (De cuidado del entorno y de seguimiento a eventos naturales adversos).

\begin{tabular}{|c|c|c|c|}
\hline Colombia & $\begin{array}{c}\text { Mapeo de iniciativas de agroecología en } \\
\text { Bogotá y sus alrededores. }\end{array}$ & $\begin{array}{l}\text { 1) Educación; 2) Semillas e } \\
\text { insumos; 3) Alimentos y } \\
\text { productos naturales. }\end{array}$ & https://agroecobogota.crowdmap.com/ \\
\hline Argentina, Bolivia, Ecuador y México & $\begin{array}{c}\text { Mapeo de experiencias vinculadas con } \\
\text { Permacultura. }\end{array}$ & $\begin{array}{l}\text { 1) Estación experimental; 2) } \\
\text { Bioconstructor; 3) Terrenos } \\
\text { disponibles; 4) Voluntariado; 5) } \\
\text { Casas en adobe; 6) Huerta } \\
\text { orgánica; 7) Minga; 8) Ecoaldea } \\
\text { conformada; 9) Ecoaldea en } \\
\text { proyecto. } \\
\end{array}$ & $\begin{array}{l}\text { https://argentinaunidaporlapermacultura. } \\
\text { crowdmap.com/ }\end{array}$ \\
\hline España & $\begin{array}{l}\text { Mapeo de experiencias de soberanía } \\
\text { alimentaria en el país de Valencia. }\end{array}$ & $\begin{array}{c}\text { 1) Producción; 2) Mercado } \\
\text { campesino; 3) Recusos; 4) } \\
\text { Consumo responsable; 5) } \\
\text { Formación, investigación y } \\
\text { publicaciones; 6) Huertas urbanas } \\
\text { comunitarias; 7) Organizaciones } \\
\text { que aborden la soberanía } \\
\text { alimentaria. } \\
\end{array}$ & https://experienciesobal.crowdmap.com/ \\
\hline $\begin{array}{l}\text { Países Bajos, Inglaterra, Estados } \\
\text { Unidos, República Dominicana, } \\
\text { Alemania, Canarias e India. }\end{array}$ & $\begin{array}{l}\text { Mapeo de experiencias de adopción de } \\
\text { vivienda alternativa Hexayurt. }\end{array}$ & $\begin{array}{l}\text { 1) Proyecto de arte; 2) Alojamiento } \\
\text { temporal; 3) Demostración de la } \\
\text { tecnología; 4) Pueblos; 5) } \\
\text { Fracasos. } \\
\end{array}$ & https://hexayurt.crowdmap.com/ \\
\hline Estados Unidos & $\begin{array}{l}\text { Mapeo de soluciones a la contamienación } \\
\text { en las cuencas bajas y los océanos. }\end{array}$ & $\begin{array}{l}\text { 1) Permeabilidad; 2) Conservación; } \\
\text { 3) Retención. }\end{array}$ & $\begin{array}{l}\text { https://oceanfriendlygardens.crowdmap.c } \\
\text { om/ }\end{array}$ \\
\hline Inglaterra & $\begin{array}{l}\text { Mapeo de efectos de las inundaciones } \\
\text { ocurridas en la costa sur de Cornwallf. }\end{array}$ & $\begin{array}{l}\text { 1) Carreteras; 2) Interrupciones de } \\
\text { tren; 3) Inundaciones; 4) Escuelas; } \\
\text { 5) Sugerencias del gobierno. }\end{array}$ & https://cornwallfloods.crowdmap.com/ \\
\hline Paquistán & $\begin{array}{c}\text { Mapeo de los incidentes ocurridos por } \\
\text { inundaciones en varias ciudades de } \\
\text { Paquistán. }\end{array}$ & $\begin{array}{l}\text { 1) Salud; 2) Agua; 3) Servicios } \\
\text { disponibles; 4) Logística y } \\
\text { magnitud de las inundaciones; 5) } \\
\text { Emergencia; 6) Abrigo; 7) } \\
\text { Seguridad; 8) Voluntarios; 9) } \\
\text { Protección, 10) Comida; 11) } \\
\text { Migración. } \\
\end{array}$ & https://pakrelief.crowdmap.com/ \\
\hline Estados Unidos & $\begin{array}{l}\text { Mapeo de los efectos del Tornado en la } \\
\text { región de San Luis. }\end{array}$ & $\begin{array}{l}\text { 1) Edificios dañados; 2) Sin } \\
\text { acceso; 3) Lesiones. }\end{array}$ & https://stlouistornado.crowdmap.com/ \\
\hline Estado Unidos y Canadá & $\begin{array}{l}\text { Mapeo de los efectos del Huracán Irene } \\
\text { en la costa Este. }\end{array}$ & $\begin{array}{l}\text { 1) Inundación; 2) Cortes de } \\
\text { energía; 3) Transporte; 4) Tiempo; } \\
\text { 5) Daños. } \\
\end{array}$ & https://irene2011.crowdmap.com/ \\
\hline Estados Unidos & $\begin{array}{c}\text { Mapeo de las incidencias de los incendios } \\
\text { forestales en Colorado. }\end{array}$ & $\begin{array}{l}\text { 1) Fuego; 2) Respuesta; 3) } \\
\text { Estructura; 4) Abrigo; 5) } \\
\text { Evacuación; 6) Emergencia. }\end{array}$ & https://coloradofires.crowdmap.com/ \\
\hline Filipinas & $\begin{array}{c}\text { Mapeo de las consecuencias del Tifon } \\
\text { Bopha. }\end{array}$ & $\begin{array}{l}\text { 1) Inundación; 2) Des lizamiento de } \\
\text { tierra; 3) Pérdida de mar; 4) } \\
\text { Inundación repentina; 5) Centros } \\
\text { de evacuación. } \\
\end{array}$ & $\begin{array}{l}\text { https://philippinedisasterwatch.crowdmap } \\
. \text { com/ }\end{array}$ \\
\hline Colombia & $\begin{array}{l}\text { Monitoreo permanente de situaciones, } \\
\text { eventos y procesos relacionados con } \\
\text { emergencias y desastres en el } \\
\text { Departamento del Chocó. }\end{array}$ & $\begin{array}{c}\text { 1) Brotes y epidemias; 2) } \\
\text { Emergencias y desastres; 3) } \\
\text { Reportes por deslizamientos; 4) } \\
\text { Reportes de necesidad médica; 5) } \\
\text { Red prestadora de servicios de } \\
\text { salud. }\end{array}$ & https://sschoco.crowdmap.com/ \\
\hline
\end{tabular}

Fuente. Elaboración propia. 


\section{APROXIMACIONES TEÓRICAS: EL AVANCE TÉCNICO DE LA GEOGRAFÍA Y LA CARTOGRAFÍA SOCIAL EN LÍNEA.}

La ciencia geográfica se ha venido redefiniendo constantemente. A través de la historia se han forjado pugnas vinculadas con la inclusión de las nociones de Espacio ${ }^{4}$, Lugar $^{5}$ y Territorio ${ }^{6}$, conceptos que han llevado consigo grandes implicaciones en el fortalecimiento de la Geografía como Ciencia.

Así las cosas, el concepto de Espacio se incorpora después de la mitad del siglo XX, como consecuencia de las fuertes críticas que recibió frente a su poco protagonismo desde el punto de vista científico, con ello adquirió la connotación de ciencia espacial. En este proceso de construcción teórica se hizo énfasis en que el problema esencial de la Geografía es estudiar las relaciones de causalidad de los fenómenos que ocurren en un espacio definido (Delgado, 2003).

La corriente filosófica que acompaño este momento de la Geografía fue el positivismo. Su nacimiento se dio a causa de la llamada revolución cuantitativa y tomaba como referente el método científico naturalista, sin embargo ésta no establecía una clara diferencia entre las metodologías para estudiar las ciencias naturales y las ciencias sociales. (Capel, 1983).

\footnotetext{
${ }^{4}$ Más aún, permite definir al espacio como una construcción social producto de procesos históricos que construye $\mathrm{n}$ y reconstruyen formas espaciales características a cada formación socio- espacial (Lerena, M. y Tomadoni, C. 1997: 725 citado por Tomadoni, 2007)

${ }^{5}$ Según GuyMercier el concepto de Lugar se puede definir como: "El lugar al menos definido como se hizo antes, no determina fundamentalmente lo que sucede en la región. En primer lugar, esto depende de lo que hemos llamado el poder intrínseco de los cuerpos y en particular, de los sujetos humanos, Sin embargo, su poder extrínseco, que define específicamente el lugar, es ciertamente ajeno al aspecto y a los matices de lo que se desarrolla allí. En efecto, si una región se distingue de otra no es simplemente por los cuerpos que contiene y por su poder extrínseco, sino también por el lugar que ella constituye a través de la manera como se ejerce el poder extrínseco, el cual determina la movilidad de los cuerpos o, dentro de un estricto enfoque de la geografía humana, el desplazamiento de los sujetos". (GuyMercier, 2009)

6 Según Tomadoni el territorio se resumen en lo siguiente: "En definitiva, el territorio no es un objeto, ni es una cosa; por el contrario, es un conjunto de relaciones complejas. La mirada transversal que puede realizarse de esta complejidad en relación a las forma s de habitar y a su utilización integral por parte de la sociedad, podría ser el ámbito de acción del saber ambiental." (Tomadoni,, 2007)
} 
Posteriormente, esta corriente epistemológica recibió bastantes críticas frente al rezago y determinismo que generaba al estudiar los fenómenos espaciales. De lo que se preocupaba la Geografía, en ese entonces, era de buscar leyes relacionadas con la organización espacial de los fenómenos. Pero, la comprensión de los mismos era un tema aún poco estudiado dentro de la ciencia Geográfica. (Delgado, 2003).

Así fue como se amplió teóricamente el horizonte de la Geografía. Pronto, gracias a las complejidades mismas de la sociedad en su conjunto, se dio nacimiento a diferentes Geografías, fundamentadas en el estructuralismo, la fenomenología, el existencialismo, entre otras corrientes epistemológicas, que enmarcaron el nacimiento de la Geografía humana.

El estructuralismo por su parte tiene una amplia relación con la teoría marxista, es decir, ven el espacio como un escenario de pugnas entre las clases sociales y se centra en dar explicación a los fenómenos desde el punto de vista económico. Mientras que la fenomenología y el existencialismo se centraron en la reivindicación del ser humano como sujeto que vive, percibe, siente y transforma del espacio. Las intencionalidades y los diferentes significados que le puede dar el ser humano al espacio se convierte en la nueva esencia de estudio de la ciencia geográfica, por ello las entrevistas a profundidad, la cartografía social, los sistemas de información geográfica participativos, se vuelven importantes metodologías cualitativas dentro de la construcción del conocimiento geográfico. La geografía humana es la rama de la ciencia geográfica encargada de buscar la comprensión de las subjetividades e intersubjetividades con las que los seres humanos moldean su Territorio. (Delgado, 2003)

De ésta forma, la geografía se centra en extraer e integrar analíticamente los aportes de las diferentes ciencias que estudian las realidades parcializadas del Espacio en el que suceden los fenómenos sociales; y con ellas crear un nuevo conocimiento que integre el estudio del por qué se formaron ciertas estructuras espaciales, procesos espaciales, flujos, redes, nodos, movimientos, jerarquías y superficies (Delgado, 2003).

Éste tipo de categorías surgen a partir de las idealizaciones, reacciones y ajustes que los seres humanos realizan a su entorno de acuerdo con sus necesidades. Por ejemplo, una estructura espacial podría ser las características de los suelos de un lugar determinado de la superficie terrestre, dependiendo de sus características físico-químicas, altura, humedad y demás propiedades biogeográficas se estructura el uso de ese suelo, es decir, si se va a dedicar al cultivo de vegetales, a la ganadería, a la urbanización o a la industria. 
De esta manera se cimenta el componente humano de la Geografía, porque se tiene que centrar en analizar el comportamiento social que construyen los procesos y estructuras socio-espaciales. Lo que interesa a la Geografía es encontrar el por qué del comportamiento espacial de los fenómenos y, por lo tanto, tendrá que dominar los conocimientos que hagan parte de la explicación integral de este fenómeno. (Buttimer, 1990; Unwin, 1995; Peet, 1998 citado por Delgado, 2003)

De acuerdo con lo anterior, un producto geográfico debe tener como referente no solo un componente cartográfico, puesto que solamente sería el equivalente a la representación espacial de un fenómeno. Detrás de éste se encuentra un problema de investigación, una justificación, un entramado teórico, unos objetivos y un desarrollo investigativo en el que muy seguramente están inmersos métodos tanto cuantitativos como cualitativos. En éstos se involucran otras ciencias como la sociología, las ciencias políticas, la antropología, la música, la pedagogía, las ciencias ambientales, entre otras. Con las cuales se busca explicar y comprender un problema geográfico para llegar a mostrar el ¿por qué?, el ¿dónde? y el ¿cómo? de sus patrones de distribución y comportamiento espacial.

\section{LA CARTOGRAFÍA SOCIAL EN LÍNEA}

La cartografía es el lenguaje de la geografía. Antes de entenderla queremos resaltar el fenómeno espacial de la segregación digital (Chaparro, 2007 y 2009) y el avance en la generación de cartografía social en línea como potenciadora de la participación ciudadana para la identificación de acciones de cambio y transformación socio-espacial.

El acceso, uso y apropiación de la tecnología y, especialmente de Internet, es diferencial en el territorio, de acuerdo con la situación geopolítica de producción de ciencia y tecnología digital de cada país a través del siglo XX. Fenómenos como las guerras han sido uno de los principales motores de producción tecno-científica -como el caso de Kenia-, por lo tanto, los países involucrados en ellas directamente tienen un nivel de acceso, uso y apropiación de conocimiento tecno-científico marcado por la generación de conocimiento de punta. En contraste, para algunos países latinoamericanos -como Colombia- involucrados con la generación de tecnología de manera tangencial, el comercio y los servicios son las maneras de acceder a la tecnología. (Chaparro, 2007)

Esta situación hace dependiente a todos los sectores de la economía y tiene bastante peso sobre la educación media y universitaria.(Gómez, 2009) 
Ahora bien, la cartografía social se define como:

...surge como nueva herramienta de planificación y transformación social. Actualmente está siendo utilizada como apoyo en procesos de organización comunitaria a través de una planificación participativa descentralizada y democrática en los que se da importancia a la participación de todos los actores locales.

Además la cartografía social de un territorio se propone como un nuevo instrumento para la construcción de conocimiento desde la participación y el compromiso social, posibilitando la transformación del mismo (Herrera, 2008).

A diferencia de la cartografía oficial en la que una entidad se encarga de escribir y marcar el territorio e manera unificada, negando la posibilidad de ver las distintas maneras de percibir e interactuar en el espacio la cartografía social trata de visibilizar los distintos actores sociales involucrados en una dinámica territorial específica. (Barrera, 2009)

La participación ciudadana en línea podría llegar a ser un instrumento que potencia la generación de conocimiento colectivo útil para la búsqueda de alternativas que ayuden a comprender y actuar frente a asuntos territoriales urgentes como la violencia por coyunturas políticas, la dinámica migratoria, la dinámica urbana, la dinámica educativa, los problemas medioambientales como los eventos naturales adversos y el cuidado del medio ambiente, entre otros.

Si bien, hay una relación de consumo en cuanto al acceso a las tecnologías e Internet en la mayoría de países latinoamericanos, esto no significa que no se pueda generar conocimiento valioso e innovador provechoso para entender las dinámicas territoriales. Lo que se necesita es visibilizar los actores territoriales y crear espacios de interacción de sus saberes con miras a crear frentes de conocimiento colectivo y emancipador. La educación tiene un fuerte compromiso territorial debe encaminarse a fortalecer la participación ciudadana en todas las escalas geográficas.

\section{POTENCIALIDADES FUNCIONALES DE LA PLATAFORMA HTTPS://AGROECOBOGOTA.CROWDMAP.COM/}

En este apartado haremos una breve descripción de las potencialidades funcionales que tiene Agroecobogotá.crowdmap.com. Las funcionalidades podemos dividirlas en cinco partes, la primera relacionada con las herramientas puestas en la vista inicial. La segunda con las potencialidades de crear reportes como impulsos de información en tiempo real. La tercera relacionada con la creación de alertas espaciales. La cuarta vinculada con la posibilidad de 
hacer análisis espaciales simples. La quinta la posibilidad de generar planos cartográficos de salida.

Figura 1.Vista inicial de la plataformahttps://agroecobogota.crowdmap.com/

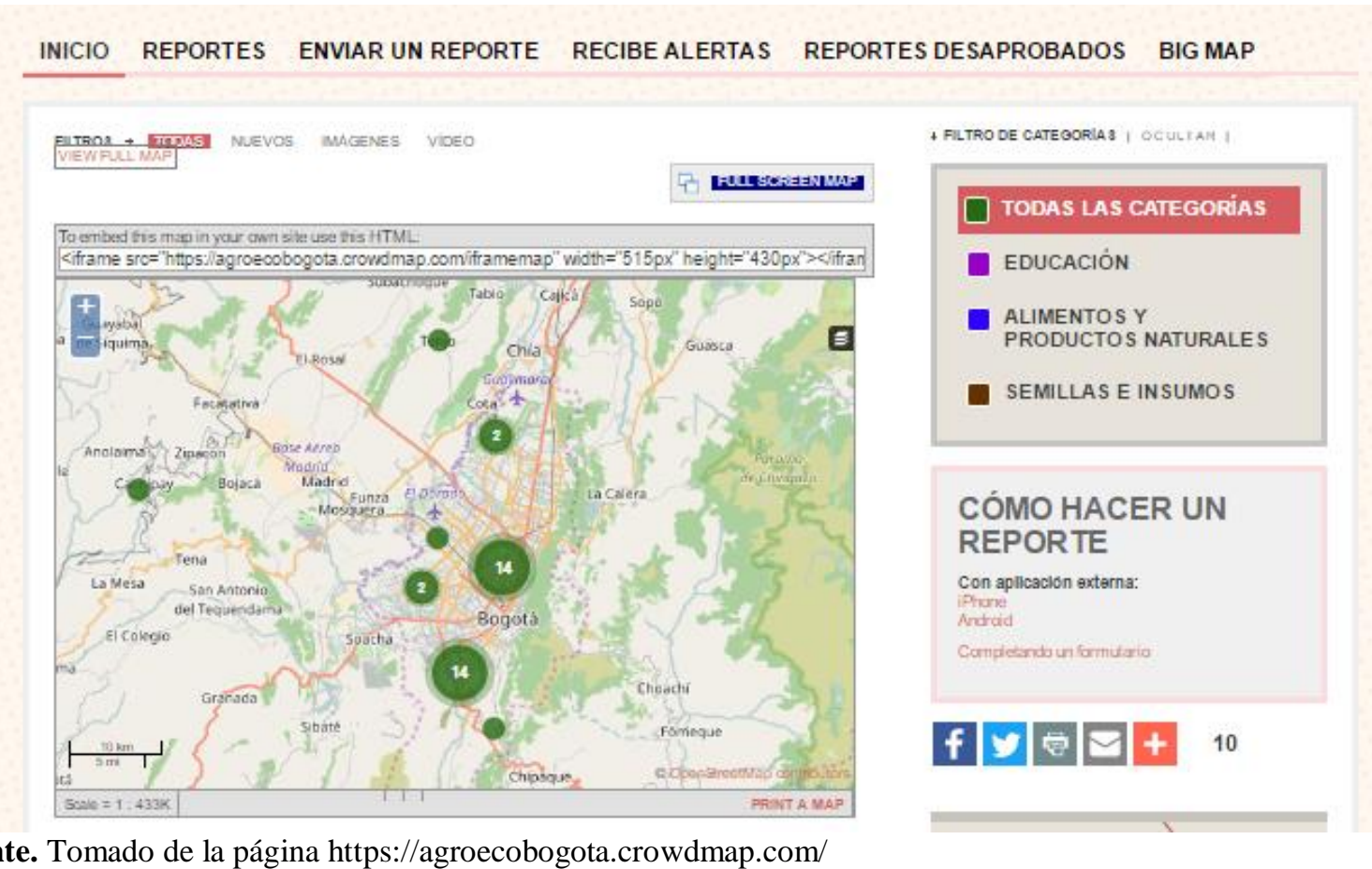

Fuente. Tomado de la página https://agroecobogota.crowdmap.com/

Existe una relación de colaboración directa entre OSM y Ushahidi, por esto la base cartográfica de la plataforma es la misma de Open Street Maps (OSM)cuya filosofía de funcionamiento es el conocimiento colectivo. La información geográfica es mundial y se muestra a escala de cuadra. En la sección que se encuentra a la izquierda de la pantalla se muestra la barra de categorías, las cuales se pueden modificar -añadir imagen, cambiar de color, quitar, sub-categorizar o añadir- de acuerdo con la intención del investigador o colectivo. Para el caso de nuestra iniciativa escogimos tres categorías: 1) educación; 2) alimentos y productos naturales; 3) semillas e insumos. La finalidad del mapeo ha sido la generación de economías locales que permitan la circulación de conocimiento ancestral en Bogotá y sus alrededores, además de fortalecer el empoderamiento territorial mediante la experiencia de aprendizaje en agroecología y permacultura que se puede adquirir en lugares alternos a la escuela -huertas caseras, colectivos agrarios, proyectos barriales, etcétera-.

La herramienta ofrece la posibilidad de compartir información web directamente con más de 20 páginas relacionadas con redes sociales de trabajo y comercio. En la parte inferior de la vista general se puede visualizar la densidad de puntos mapeados a través del tiempo, 
indicando los picos en los que más han llegado reportes y, los tiempos en los que el mapeo no ha contado con mucho movimiento. También hay espacios en donde se pueden compartir blogs o páginas web relacionadas con las temáticas de la plataforma, esta parte recibe el nombre de portal de noticias principales.

En la parte superior, está la herramienta más importante: Enviar reporte. La función principal de este botón es enviar al ciudadano a la plantilla en donde puede generar información geográfica específica y alimentada colaborativamente. La secciones del reporte son seis en total: 1) Titulo del reporte: corresponde al nombre proyecto o colectivo. 2) Descripción: habilita en lenguaje de texto de la experiencia. Tiene un máximo de 1.500 caracteres. 3) La selección de la categoría de mapeo a la que pertenece. También se pueden crear preguntas específicas para rastrear un fenómeno en tiempo real. 4) Información de contacto -nombre, correo, teléfono- hay algunos mapeos que al momento de validar ${ }^{7}$ la información la retiran por asuntos de seguridad. 5) La ubicación del punto en donde se maneja el lenguaje vectores decir, la posibilidad de crear sea un punto, línea o polígono en el mapa. Se cuenta con localizador de ubicación que permite emplear tanto coordenadas geográficas, como el nombre concreto del lugar. 6) La última sección es tal vez la más importante porque permite añadir archivos de enlace fuente de noticias, de imagen y de vídeo -sin límite de cantidad y con libertad de formato electrónico-. Al terminar de completar la información se pulsa enviar. La plataforma almacena la información y solo la muestra cuando ha finalizado el proceso de verificación.

\footnotetext{
${ }^{7}$ La plataforma ofrece dos maneras de validar la información: Una obedece a un filtro temático en donde se revisa que el colectivo en mención esté en funcionamiento y la información sea verídica, y el otro es un filtro técnico en donde se verifica la georreferenciación de los puntos y la claridad de los datos dados en el reporte.
} 
Figura 2. Vista de la sección de Reporte.

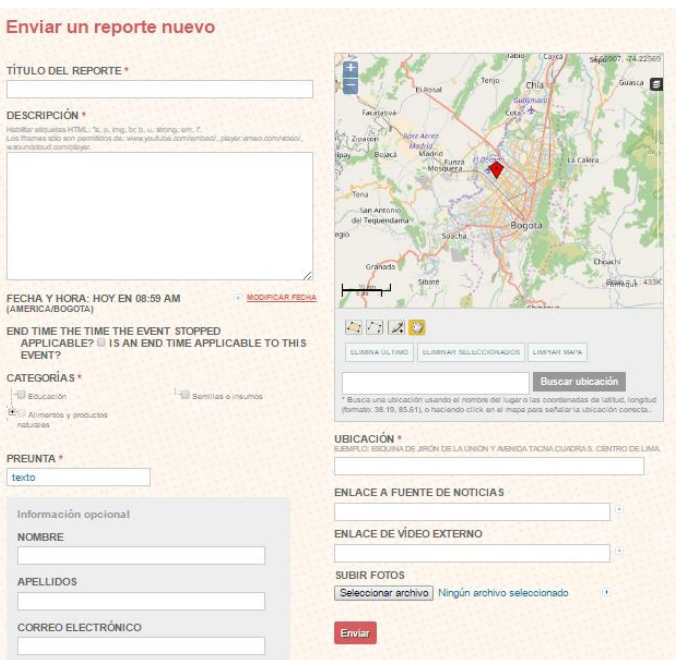

Figura 3. Vista la sección de alertas espaciales.

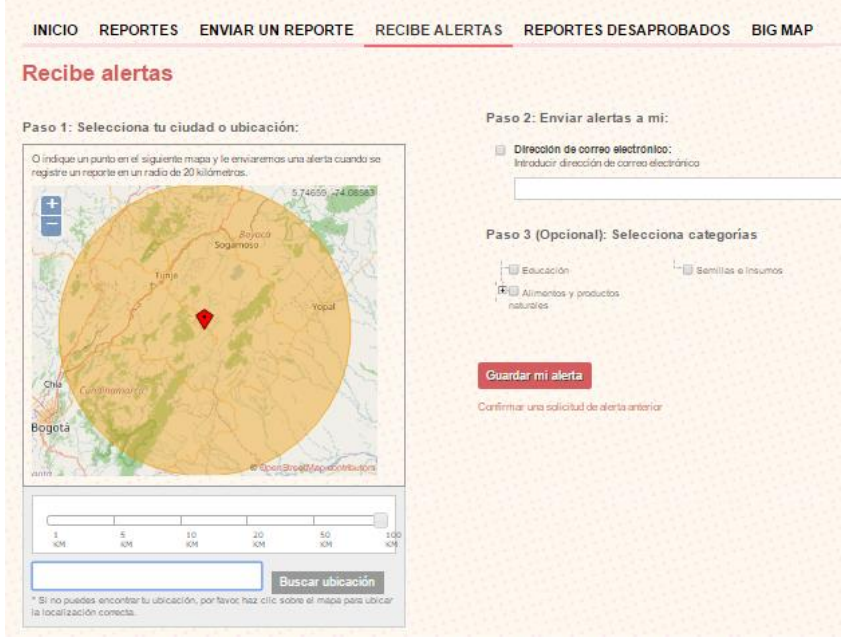

Fuente. Tomado de la página https://agroecobogota.crowdmap.com/

Agroecobogota, permite la creación de alertas espaciales de información geográfica específica. Es decir, brinda la posibilidad de asociar a la cuenta de correo electrónico los reportes que se hagan en un radio que puede ir desde un kilómetro hasta cien kilómetros a la redonda. https://agroecobogota.crowdmap.com/En la sección se encuentra la barra horizontal en donde se escribe la cuenta de correo electrónico, luego de ello las categorías de interés. Al guardar la alerta, inmediatamente empieza a replicarse la información al correo inscrito de los reportes hechos en el espacio de escala escogido y la categoría seleccionada.

La cuarta sección involucra la posibilidad de hacer análisis espaciales simples empleando operadores booleanos AND y OR:

- Utilizar el operador AND para localizar registros que contengan todos los términos de búsqueda especificados. Por ejemplo, si se busca por "perros AND gatos", localiza registros que contengan todos los términos especificados.

- Utilizar el operador OR para localizar registros que contengan cualquiera o todos los términos especificados. Por ejemplo, si se busca por "perros OR gatos", la biblioteca-e localiza registros que contengan el primer término o el segundo (Ushahidi, 2012)

Dando como resultado, algunas discriminaciones espaciales que pueden llegar a ayudar a tomar decisiones de tipo económico, por ejemplo. Esta herramienta está en la sección Big map. Y se complementa con el tablero de seguimiento temporal de los reportes hechos, el tamaño de los puntos y la posibilidad de habilitar o inhabilitar la agrupación espacial de los mismos, o sea la función clustering. 
Figura 4. Vista la sección relacionada con la salida gráfica del mapa.
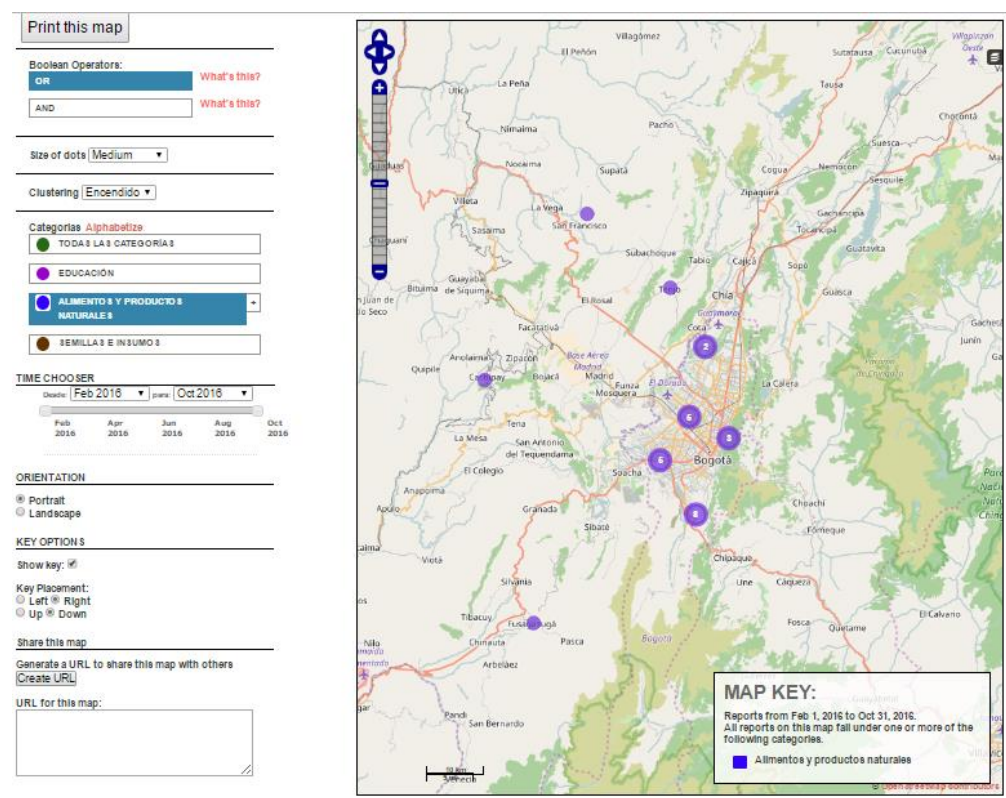

Fuente. Tomado de la página https://agroecobogota.crowdmap.com/

Por último, en la parte inferior izquierda del mapa aparece el hipervínculo printmap. Este direcciona a la persona a la vista de la Figura x, en donde se pueden hacer acciones de edición para realizar salidas gráficas del mapa construido colaborativamente. Es una herramienta muy útil para el trabajo colectivo, puesto que brinda la posibilidad de complementar el mapa electrónico con propuestas analógicas que se pueden llevar a cabo en ambientes analógicos. Las secciones de esta parte de la plataforma vinculan análisis espaciales simples, discriminación temporal de los reportes hechos, edición de la leyenda del mapa y la posibilidad de creación de una dirección electrónica específica del mapa editado, asunto muy útil para la toma de decisiones en ámbitos territoriales.

\section{COMENTARIOS FINALES}

Consideramos el mapeo en tiempo real como un importante avance en la creación cartográfica digital. El hecho de que las mismas ciudadanías puedan mostrar su manera de percibir el territorio de acuerdo a una lógica de vida es un lenguaje geográfico que hasta ahora se asoma en las dinámicas teóricas de la geografía.

La posibilidad de construir en tiempo real hace que se tenga una visión del problema mucho más sistematizada y un acercamiento a los fenómenos un poco más exacto. Consideramos que la tecnología Crowdmap es una muy buena herramienta para emancipar poblaciones y tratar de entender las coyunturas territoriales presentes. 
Queremos resaltar que contamos con un total de cuarenta puntos mapeados en la plataforma, distribuidos de la siguiente manera: veintisiete iniciativas de educación agroecológica, veintiocho iniciativas relacionadas con la producción de alimentos y productos naturales y veintidós relacionadas con la comercialización de semillas e insumos orgánicos.

La iniciativa de mapeo se encuentra vigente y en línea permanente. Contamos con una página en Facebook llamada "mapeo agroecobogota" en donde cada vez son más los seguidores... En ella se encuentran diferentes eventos relacionados con la soberanía alimentaria y el aprendizaje agroecológico en Bogotá y sus alrededores. La plataforma puede llegar a tener incidencia internacional. Tiene la potencialidad tecnológica brindada por Ushahidi.

Esta iniciativa es el primer mapeo en tiempo real que involucra los saberes agroecológicos para que la misma población intercambie sus saberes en la producción de alimentos y el buen vivir. Se quiere mostrar como una iniciativa que busca el intercambio de experiencias desde una lógica horizontal de aprendizaje y participación ciudadana continua.

\section{BIBLIOGRAFÍA}

Arteaga Botello, N., \& Valdés Figueroa, J. (2010). Contextos socioculturales de los feminicidios en el Estado de México: nuevas subjetividades femeninas. Revista mexicana de sociología, 72(1), 5-35.

Buttimer, A. (1990). Geography, Humanism, and Global Concern1. Annals of theAssociation of American Geographers, 80(1), 1-33.

Campo, V. M. G., Ríos, C. M. D., \& Giraldo, J. E. C. (2009). El puente está quebrado...: aportes a la reconstrucción de la educación media en Colombia. Univ. Nacional de Colombia.

Herrera, J. (2008). Cartografía social. Recuperado el, 16.

Herreño Lopera, (2012). Análisis de la inestabilidad política como obstáculo al desarrollo en el marco de los Objetivos de Desarrollo del Milenio. Estudio de caso Kenia (2002-2008).

Lobatón, S. B. (2009). Reflexiones sobre Sistemas de Información Geográfica Participativos (SIGP) y cartografía social. Cuadernos de geografía, (18), 9.

Mahecha, O. D. (2003). Debates sobre el espacio en la geografía contemporánea. Univ. Nacional de Colombia. 
Mendivelso, J. C. (2007). La segregación digital en América Latina y el Caribe: reflejo de las inequidades sociales y la dependencia tecnológica. Scripta Nova: revista electrónica de geografía y ciencias sociales, 11 .

Mendivelso, J. C. (2009). Un mundo digital. Cibergeografía, bits y nuevas tecnologías de la información y la comunicación a inicios del siglo XXI (Doctoral dissertation, Tesis doctoral dirigida por el Dr. Horacio Capel, Universidad de Barcelona, Departamento de Geografía Humana).

Mercier, G. (2009). Hacia una teoría del lugar. Geografía humana. Lecturas en teoría de la geografía, 21-40.

Okolloh, O. (2012). Ushahidi, or 'testimony': Web 2.0 toolsforcrowdsourcing crisis information. Participatorylearning and action, 59(1), 65-70.

Peet, R. (1998). Modern geographicalthoughtBlackwell.

Sáez, H. C. (1983). Positivismo y antipositivismo en la ciencia geográfica: el ejemplo de la geomorfología. Univ. Barcelona.

Tomadoni, C. (2007). A propósito de las nociones de espacio y territorio. Gestión y ambiente, 10(1), 53-66.

Unwin, T. H. (1995). El lugar de la Geografía. Cátedra. 